\title{
Optimal cloud assistance policy of end-edge-cloud ecosystem for mitigating edge distributed denial of service attacks
}

Teng Li (D)

\begin{abstract}
Edge computing has become a fundamental technology for Internet of Things (loT) applications. To provide reliable services for latency-sensitive applications, edge servers must respond to end devices within the shortest amount of time possible. Edge distributed denial-of-service (DDoS) attacks, which render edge servers unusable by legitimate loT applications by sending heavy requests from distributed attacking sources, is a threat that leads to severe latency. To protect edge servers from DDoS attacks, a hybrid computing paradigm known as an end-edge-cloud ecosystem provides a possible solution. Cloud assistance is allowed with this architecture. Edge servers can upload their pending tasks onto a cloud center for a workload reduction when encountering a DDoS attack, similar to borrowing resources from the cloud. Nevertheless, before using the ecosystem to mitigate edge DDoS attacks, we must address the core problem that edge servers must decide when and to what extent they should upload tasks to the cloud center. In this study, we focus on the design of optimal cloud assistance policies. First, we propose an edge workload evolution model that describes how the workload of the edge servers change over time with a given cloud assistance policy. On this basis, we quantify the effectiveness of the policy by using the resulting overall latency and formulate an optimal control problem for seeking optimal policies that can minimize such latency. We then provide solutions by deriving the optimality system and discuss some properties of the optimal solutions to accelerate the problem solving. Next, we introduce a numerical iterative algorithm to seek solutions that can satisfy the optimality system. Finally, we provide several illustrative numerical examples. The results show that the optimal policies obtained can effectively mitigate edge DDoS attacks.
\end{abstract}

Keywords: Edge-cloud computing, Distributed denial-of-service attacks, Cloud assistance, Optimal control, Pontryagin minimum principle

\section{Introduction}

With the development of microcomputer technology, Internet-of-Things (IoT) devices have been widely deployed for data collection $[1,2]$. However, IoT devices have difficulty supporting resource-intensive applications owing to energy and computational constraints. To tackle this issue, edge computing [3, 4], a computation paradigm that allows IoT tasks to be processed at the edge of the

Correspondence: cqcetliteng@163.com

Artificial Intelligence and Big Data College, Chongqing College of Electronic Engineering, Chongqing, China
Internet, has been proposed for efficient computation offloading. In recent years, this technology has been applied to various applications, including smart grids [5], connected health [6], and connected automated vehicles [7].

Because edge computing has become a fundamental technology for IoT applications, and edge servers (ESs) must have strong security for providing reliable services. Among common cyber threats to ESs, distributed denialof-service (DDoS) attacks $[8,9]$ can lead to severe results. As the name suggests, a DDoS attack renders ESs unusable by legitimate IoT applications by sending heavy resource-

\section{Springer Open}

(C) The Author(s). 2021 Open Access This article is licensed under a Creative Commons Attribution 4.0 International License, which permits use, sharing, adaptation, distribution and reproduction in any medium or format, as long as you give appropriate credit to the original author(s) and the source, provide a link to the Creative Commons licence, and indicate if changes were made. The images or other third party material in this article are included in the article's Creative Commons licence, unless indicated otherwise in a credit line to the material. If material is not included in the article's Creative Commons licence and your intended use is not permitted by statutory regulation or exceeds the permitted use, you will need to obtain permission directly from the copyright holder. To view a copy of this licence, visit http://creativecommons.org/licenses/by/4.0/. 
consuming requests from distributed attacking sources. Because of the inherent vulnerabilities of IoT devices [10-12], attackers can infect numerous IoT devices without much effort and offload heavy tasks onto the ESs. Consequently, the ESs will likely crash, and the response time of the computation tasks will become too long to ensure reliable services for latency-sensitive applications. For convenience, we refer to this type of attack as an edge DDoS attack. Figure 1 shows a diagram of the relevant scenario. Therefore, defending against edge DDoS attacks has become an essential issue in the field of edge computing $[13,14]$.

\section{Motivation}

To mitigate edge DDoS attacks, a hybrid computing paradigm known as an end-edge-cloud (EEC) ecosystem [15-18] provides a possible solution, converging the designs of cloud $[19,20]$ and edge $[3,4]$ computing. As shown in Fig. 2, such an ecosystem includes a super-capacity cloud center, numerous constrainedcapacity ESs, and a large number of low-capacity end devices. Computation tasks produced by the end side are processed at either the edge or cloud side. The workflow can be described as follows: First, IoT tasks arrive at a local access point (AP) that connects the end and edge sides. Then, with the control of a load balance scheme, the local AP dispatches these tasks within the interconnecting network of the ESs. Next, at an ES, some arriving tasks will be processed immediately, whereas some will have to wait for computational resources. If the resources of an ES are insufficient, the ES can proactively outsource a portion of pending tasks onto the cloud center, similar to borrowing resources from the cloud side. Under this mechanism, if an edge DDoS attack occurs, the cloud side will act as an emergency resource to relieve the workload of the edge.

Nevertheless, before implementing the above blueprint, we must address the core problem, namely, when and to what extent an ES should upload its pending tasks onto the cloud center, thereby minimizing the impact caused by DDoS attacks. We refer to this as the cloud assistance (CA) problem, and refer to any solution as the CA pol$i c y$. Solving the CA problem is complex. On the one hand, with the constrained resource of an ES, a lower assistance level will result in a higher computational latency; on the other hand, because the bandwidth is a bottleneck of the network core, a higher assistance level will result in a higher communication latency. To provide reliable services, ESs must respond to the end devices within the shortest possible time. Therefore, an effective CA policy should account for both computation and communication delays and minimize the overall latency.

\section{Contributions}

In this study, a design for using the EEC ecosystem to mitigate edge DDoS attacks was developed. In particular, we address the CA problem. Our contributions are as follows:

- We propose a novel dynamic edge workload model that incorporates the changing workload of the ESs under the effects of a DDoS attack, a load balance scheme, and a CA policy. On this basis, we evaluate the effectiveness of the CA policy by quantifying the resulting overall latency, which consists of computation and communication delays. Then, to seek optimal CA policies, we formulate an optimal control problem, where a solution represents a policy

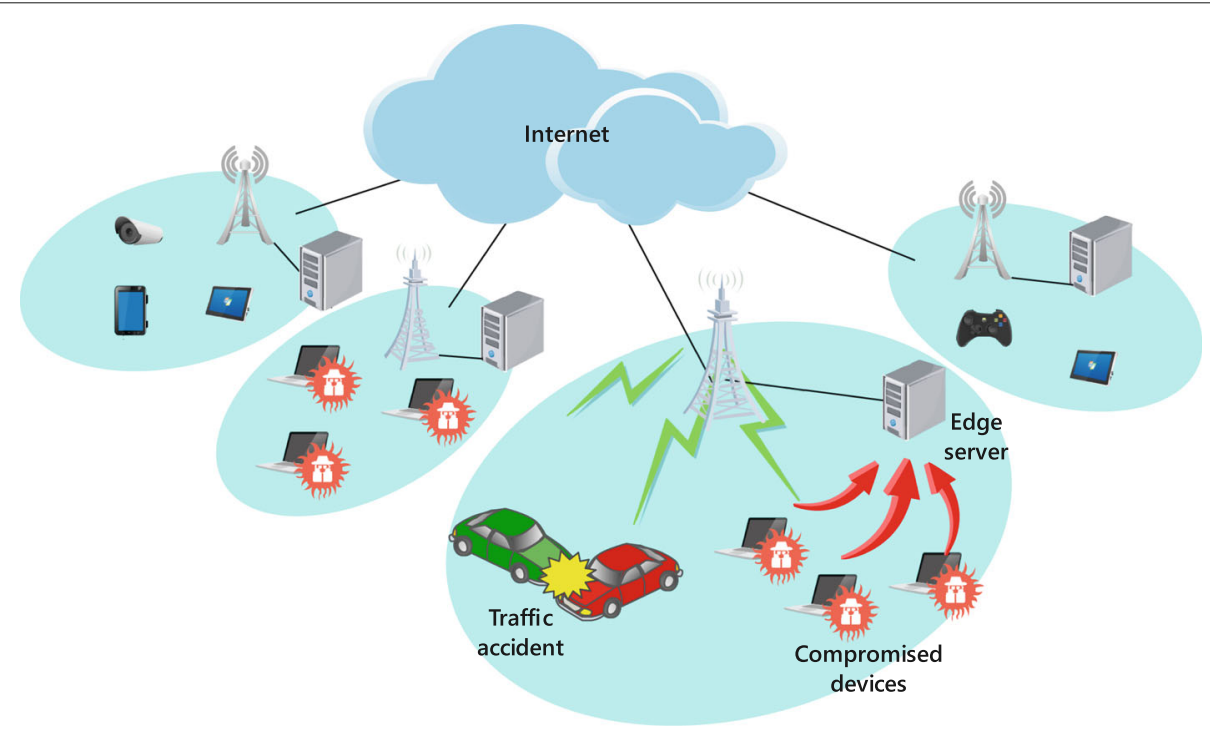

Fig. 1 A scenario of edge DDoS attacks 


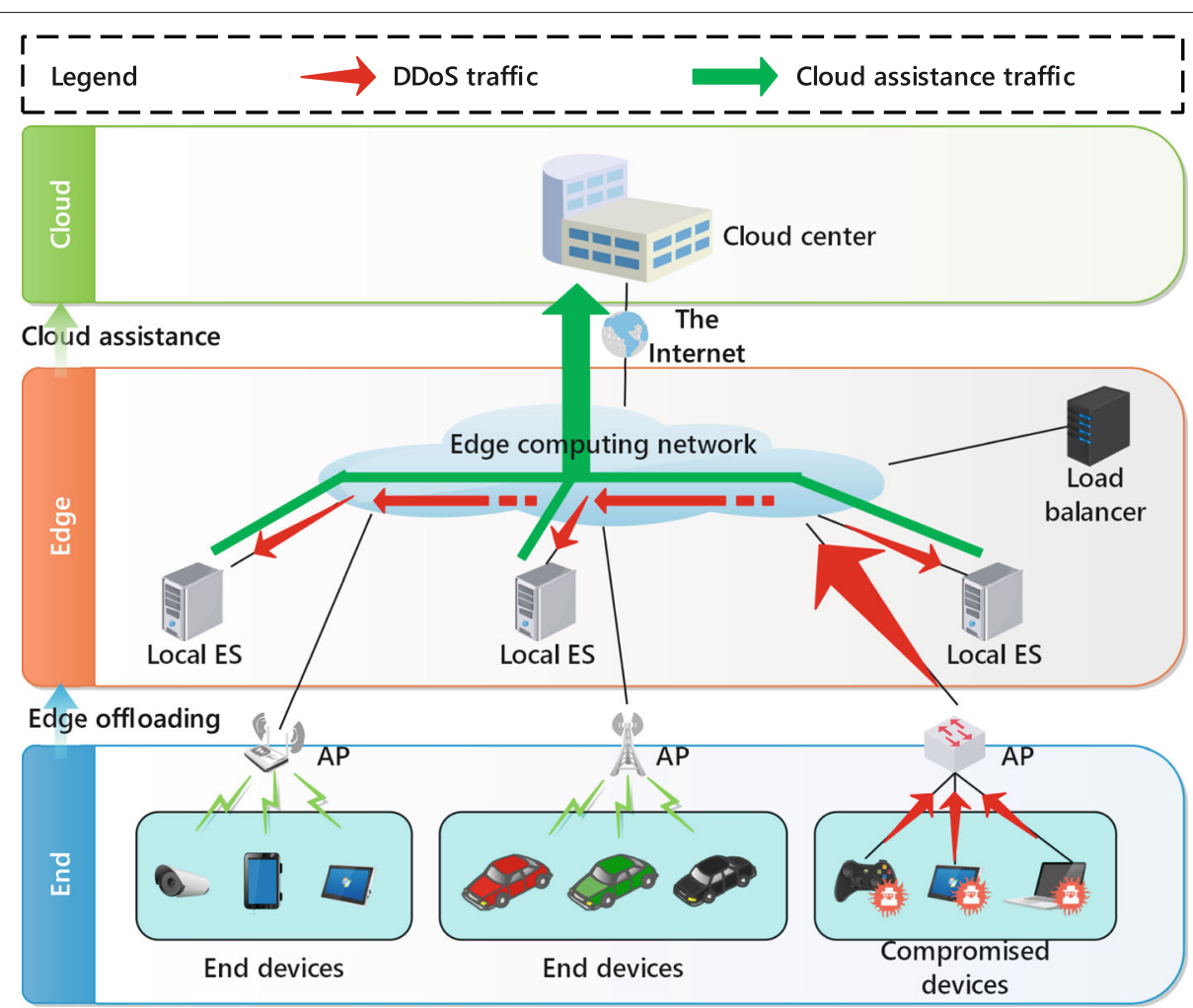

Fig. 2 A diagram of how an EEC ecosystem can mitigate an edge DDoS attack. The DDoS traffic, on the one hand, will be distributed among local edge servers owing to the load balancer, on the other hand, will be uploaded onto the cloud center owing to the cloud assistance mechanism

and the objective functional stands for the resulting overall latency.

- Based on Pontryagin's minimum principle [21], we derive the optimality system for solving the formulated optimal control problem, which provides a set of necessary optimality conditions. To accelerate the problem solving, we then discuss some properties of the optimal solutions. To solve the problem numerically, we introduce an iterative algorithm based on the forward-backward sweep method [22] to find possible optimal solutions that satisfy the optimality system.

- We provide several numerical examples to illustrate the optimal CA policies. The results show that the optimal policies obtained can effectively mitigate the impact of DDoS attacks.

The remainder of this paper is organized as follows. "Related work" section reviews related studies and outlines our innovations. "System modeling and problem formulation" section is devoted to system modeling and problem formulation. "Solution" section focuses on solutions. Several numerical examples are provided in "Illustrative examples" section. The remainder of this paper is organized as "Conclusion" section.

\section{Related work}

In this section, we discuss related studies and outline our innovations. First, we review the efforts to mitigate DDoS attacks. Second, we discuss the relationship between our modeling approach and previous research on computing offloading modeling.

\section{Mitigation of DDoS attacks}

A DDoS attack refers to any type of malicious operation that renders a server unusable by sending resourceconsuming requests. There are two major mitigation approaches.

The first approach is devoted to the detection and elimination of malicious requests. Researchers in this field believe that there must be some differences between normal and malicious operations. On this basis, they analyzed the features of different types of attacks and developed corresponding protective methods, including behaviorbased approaches [23-25] for detecting abnormal request sequences, traffic-based methods [26, 27] for detecting abnormal data flows, and request-based methods [28-30] for detecting abnormal packets. However, the detectability of malicious requests remains to be confirmed because it is difficult to determine whether a sophisticated attack can forge malicious requests that are extremely similar 
to the normal request. In fact, it was shown that bots can forge the behaviors of human users and break some commonly used security protections with the aid of artificial intelligence technology [31-34]. Therefore, detectionbased methods seem to require more fault tolerance to defend against advanced DDoS attacks.

The other approach, which does not have to rely on the detectability of malicious requests and hence has a good fault tolerance for protecting against general DDoS attacks, is to absorb the high-volume attacking traffic with larger-capacity machines. We refer to this as a capacitybased approach. An important technique in this field is to use the content delivery network (CDN) as an emergency resource to handle malicious operations [35-39]. When an attack occurs, idle CDN nodes copy the required static contents as a cache from the origin ES and use the cache to help respond to malicious requests, without regard to the attack detail. With this universality, the CDN-based method has become a popular commercial solution for DDoS attack mitigation. However, when the attack is designed to request different dynamic contents, the CDN will have to continuously copy related content from the origin ES. In this case, the origin of the ES workload cannot be effectively reduced.

The use of an EEC ecosystem to mitigate edge DDoS attacks is a possible solution that follows the capacitybased approach. With this method, a super-capacity cloud center is used as an emergency resource to help handle enormous malicious requests on the edge side. Unlike the CDN-based method, the ecosystem is a distributed cluster in which any single component can function as an independent origin server. Hence, when malicious requests are distinct or dynamic, the ecosystem can protect ESs using cloud resources. However, there are no references that can inform the design of related policies for implementation, and the CA problem remains to be solved.

In this paper, we focus on the design of effective CA policies that will help develop the design of an ecosystem to mitigate edge DDoS attacks. To the best of our knowledge, this is the first study to make such an attempt.

\section{Relations between our modeling approach and computing offloading models}

A computing offloading problem is about how to upload the tasks generated from the end side onto the edge or cloud side.

A common methodology to address edge computing offloading problems includes the following three steps. First, specify a static controllable decision variable. Second, formulate a set of performance indice (e.g., the energy consumption) for the static decision variable, and reduce a computing offloading problem as a programming problem (e.g., the nonlinear mixed integer programming problem). Third, investigate the properties of the assignment problem and design a new algorithm with the properties. See [40-42] for certain examples. For convenience, we refer to all the computing offloading models of this methodology as the static offloading models. In a static offloading model, the decision variable will not change over time, and the offloading process is usually considered to be an instantaneous event. Static offloading models are not suitable for the study of DDoS mitigation, because a DDoS attack usually takes palce over a long period.

Some studies consider the dynamic process of computing offloading. See [43-47] for certain examples. We refer to all the related models as the dynamic offloading models. In a dynamic offloading model, new tasks will be continuously generated from the end side over time. Accordingly, the decision variable is a sequence of time, and the change of the decision variable will lead to the evolution of the offloading process. Our modeling approach is similar to the dynamic offloading modeling technique, because a DDoS attack can be also regarded as a large-scale task concurrent from the perspective of computing offloading.

However, to our best knowledge, existing dynamic offloading models can not be directly applied in our study. There are two reasons. First, load balance is an important part of an EEC ecosystem, but the influence of different load balance schemes is seldom considered in offloading models. Second, we can hardly see any dynamic offloading model that characterizes the cloud assistance process of an EEC ecosystem. Therefore, we have to propose a novel workload evolution model.

\section{System modeling and problem formulation}

In this section, we are devoted to designing an effective CA policy for mitigating edge DDoS attacks. First, in order to capture the effect of CA policies, we propose a novel workload evolution model for ESs, accounting for different load balance schemes applied to the edge computing network. Second, we quantify the overall latency caused by computation and communication delays. Then, we formulate an optimal control problem for seeking optimal policies.

\section{A evolution model of edge workload distribution}

Let us start by introducing some notations. As we mentioned before, the core of an EEC ecosystem consists of a distributed edge computing network and a cloud center. Suppose that the edge network is composed of $M$ ESs, each of which has a unique ID labeled by $s_{1}, s_{2}, \ldots, s_{M}$. Because load balance is available in the network, we need to model the relations among all ESs in terms of computation forwarding. Let $S=\left\{s_{1}, s_{2}, \ldots, s_{M}\right\}$ denote the set of all ESs. Denote by $e_{i j}$ the edge from $s_{i}$ to $s_{j}$ if server $s_{i}$ is able to forward its computation tasks to server $s_{j}$. Let $E$ be the set of all such logical edges. Then, we use a graph 
$G=(S, E)$ to represent the computation forwarding relations among ESs. For convenience, denote by $A_{M \times M}$ the adjacency matrix of $G$, where $A_{i j}=1$ or 0 according to whether $e_{i j} \in E$ or not. In particular, let $A_{i i}=0$ for all $i=1,2, \ldots, M$. Also, we define a modified matrix $B_{M \times M}$ where $B_{i j}=A_{i j}, i \neq j$, and $B_{i i}=1, i=1,2, \ldots, M$.

Consider a small time horizon $t \in[0, T]$, in which a developed policy makes effect. On measuring the impact of an DDoS attack, we denote by $\alpha_{i}(t)$ the increase rate of new computation tasks, which can be obtained by estimation or prediction with the help of historical data. Suppose that each ES has a infinite-length task queue, and denote by $w_{i}(t) \in[0, \infty]$ the workload of server $s_{i}$ at time $t$. We refer to the $M$-dim function $w(t)=$ $\left(w_{1}(t), w_{2}(t), \ldots, w_{M}(t)\right), 0 \leq t \leq T$, as the workload distribution of the edge computing network. Denote by $u_{i}(t) \in[0,1]$ the related assistance rate at time $t$, which controls the proportion of pending tasks that will be transferred from server $s_{i}$ to the cloud. Then, the CA policy is represented by the $M$-dim function $u(t)=$ $\left(u_{1}(t), u_{2}(t), \ldots, u_{M}(t)\right)$. Assume that all CA policies are Lebesgue integrable, and let $\Omega$ be the set of all Lebesgue integrable functions defined on the time horizon $[0, T]$, whose value space at any time is $[0,1]^{M}$. Then, we refer to $\Omega$ as the admissible set of CA policies. Accounting for the influence of load balance, we denote by $f_{i j}(t) \in$ $[0,1]$ the computation forwarding ratio at time $t$, which represents the proportion of pending tasks that will be transferred from server $s_{i}$ to $s_{j}$, provided that load balance is available between the two ESs. Because of computation conservation, we have $\sum_{j=1}^{M} B_{i j} f_{i j}(t)=1$ for any time $t$.

Next, we need to model how the workload distribution will evolves over time under a given CA policy. According to the workflow of the EEC ecosystem, the workload of an ES can be affected by three factors: (a) the computation increase due to the DDoS attack, (b) the computation forwarding due to the load balance scheme, and (c) the computation offloading due to the cloud assistance. On the one hand, the workload of a server would increase due to receiving new computation tasks from end devices and peer ESs. On the other hand, the workload of a server would decrease due to uploading computation tasks to the cloud. The following theorem provides the dynamic system of the workload distribution.

Theorem 1 The workload distribution $w(t)$ evolves according to the following dynamic system:

$$
\left\{\begin{array}{c}
\dot{w}_{i}(t)=-u_{i}(t) w_{i}(t)+\alpha_{i}(t) f_{i i}(t)+\sum_{j=1}^{M} A_{j i} f_{j i}(t) \alpha_{j}(t), \\
0 \leq t \leq T, i=1,2, \ldots, M \\
w(0)=w_{0}
\end{array}\right.
$$

where $w_{0} \in R^{M}$ represents the initial workload distribution.

Proof Let $\Delta t$ be a variation of time. For any time $t$,

- Due to the computation increase of the locality, the workload of $s_{i}$ increases at the rate of $\alpha_{i}(t)$;

- Due to the computation receiving from other servers, the workload of $s_{i}$ increases at the rate of $\sum_{j=1}^{M} A_{j i} f_{j i}(t) \alpha_{j}(t)$

- Due to the computation forwarding to other servers, the workload of $s_{i}$ decreases at the rate $\alpha_{i}(t) \sum_{j=1}^{M} A_{i j} f_{i j}(t)$;

- Due to cloud assistance, the workload of $s_{i}$ decreases at the rate of $w_{i}(t) u_{i}(t)$.

Therefore, it follows that

$$
\begin{aligned}
w_{i}(t+\Delta t) & =w_{i}(t)+\alpha_{i}(t) \Delta t-w_{i}(t) u_{i}(t) \Delta t-\alpha_{i}(t) \sum_{j=1}^{M} A_{i j} f_{i j}(t) \cdot \Delta t \\
& +\sum_{j=1}^{M} A_{j i} f_{j i}(t) \alpha_{j}(t) \cdot \Delta t .
\end{aligned}
$$

As $\sum_{j=1}^{M} B_{i j} f_{i j}(t)=1$, we have

$$
\begin{aligned}
\dot{w}_{i}(t) & =\lim _{\Delta t \rightarrow 0} \frac{w_{i}(t+\Delta t)-w_{i}(t)}{\Delta t} \\
& =\alpha_{i}(t)-w_{i}(t) u_{i}(t)+\alpha_{i}(t) f_{i i}(t)+\sum_{j=1}^{M} A_{j i} f_{j i}(t) \alpha_{j}(t) .
\end{aligned}
$$

The proof is completed.

With this dynamic system, we can predict the workload of any server at any time, which provides the foundation for quantifying the overall latency caused by a specific CA policy. We refer to this dynamic system as the workload distribution evolution model, also short for the workload model.

\section{Examples of load balance schemes}

In the previous subsection, we proposed a workload model for capturing the workload distribution dynamics of the distributed edge computing system, where we denoted by the function $f$ the computation forwarding ratio that represents the influence of different load balance schemes. In this subsection, we give examples of charactering such type of functions.

In the first example, we consider the Round Robin scheme [48], one of the most commonly-used load balance algorithms. In the RR scheme, a controller equally dispatches newly-received computation tasks among ESs 
in spite of their current workload distribution. The forwarding ratio from server $s_{i}$ to $s_{j}$ can be expressed as

$f_{i j}^{R R}(t)=\frac{1}{\sum_{k=1}^{M} B_{i k}}, \quad i=1, \ldots, M, j=1, \ldots, M, 0 \leq t \leq T$.

In the second example, we consider the Least Connection scheme [49]. Different from the RR scheme, the LC scheme takes into account the current workload distribution of ESs. Among all servers, the one that is with a higher workload will receive fewer tasks; on the contrary, the one that is with a lower workload will receive more tasks, so that the workload of each server can be maintained at the same level as much as possible. The forwarding ratio from server $s_{i}$ to $s_{j}$ can be expressed as

$$
\begin{gathered}
\bar{f}_{i j}^{L C}(t)=\frac{\frac{\sum_{k=1}^{M} B_{i k} w_{k}(t)}{w_{j}(t)}}{\sum_{l=1}^{M} B_{i l} \cdot \frac{\sum_{k=1}^{M} B_{i k} w_{k}(t)}{w_{l}(t)}}=\frac{\frac{1}{w_{j}(t)}}{\sum_{l=1}^{M} B_{i l} \cdot \frac{1}{w_{l}(t)}} \\
i=1, \ldots, M, j=1, \ldots, M, 0 \leq t \leq T .
\end{gathered}
$$

As the workload of a server can be zero, let $\epsilon$ be a small number and let the function $f_{i j}$ be approximated as

$$
f_{i j}^{L C}(t)=\frac{\frac{1}{w_{j}(t)+\epsilon}}{\sum_{l=1}^{M} B_{i l} \cdot \frac{1}{w_{l}(t)+\epsilon}}, \quad i=1, \ldots, M, j=1, \ldots, M, 0 \leq t \leq T .
$$

\section{An optimal control problem}

In the previous subsections, we proposed a workload model and gave some examples of charactering different load balance schemes. In this subsection, we are devoted to formulating an optimal control problem for seeking optimal CA policies.

First, we need to quantify the overall latency caused by a given CA policy. In practice, as we assume that the cloud is capability-infinite, the overall latency comes from three parts: (a) the communication latency of cloud assistance, (b) the communication latency of edge load balance, and (c) the computation latency of edge computing.

First, suppose that at any time the communication delay of transferring $z$ computation tasks from the edge to the cloud increases at the rate $h(z)$. Then, given the initial workload distribution $w_{0}$ and the CA policy $u(t)$, the expected total communication delay of transferring tasks from the edge to the cloud during the time horizon $[0, T]$ is

$$
D_{B}\left(w_{0}, u\right)=\int_{0}^{T} \sum_{i=1}^{M} h\left(u_{i}(t) w_{i}(t)\right) \mathrm{d} t .
$$

Second, as all computation forwarding is one-hop, the total communication delay among ESs is determined by the number of forwarded tasks. Let $d_{i j}$ be the average delay of forwarding one task from server $s_{i}$ to $s_{j}$. Note that the task forwarding from $s_{i}$ to $s_{j}$ and that from $s_{j}$ to $s_{i}$ can not be offset. Then, given the initial workload distribution $w_{0}$ and the CA policy $u(t)$, the expected total communication delay of forwarding tasks among ESs during the time horizon $[0, T]$ is

$$
D_{C}\left(w_{0}, u\right)=\int_{0}^{T} \sum_{i=1}^{M} \sum_{j=1}^{M} A_{i j} f_{i j}(t) \alpha_{i}(t) d_{i j} \mathrm{~d} t .
$$

Third, let $g(z)$ denote the overall computation delay per unit time when the workload of a server is $z$, where $g$ is an increasing function of $z$ and $g(0)=0$. Then, given the initial workload distribution $w_{0}$ and the CA policy $u(t)$, the expected total computation delay at the edge is

$$
D_{P}\left(w_{0}, u\right)=\int_{0}^{T} \sum_{i=1}^{M} g\left(w_{i}(t)\right) \mathrm{d} t .
$$

To summarize, given the initial workload distribution $w_{0}$, the overall latency caused by the CA policy $u(t)$ is

$$
\begin{aligned}
J\left(w_{0}, u\right)= & D_{B}\left(w_{0}, u\right)+D_{C}\left(w_{0}, u\right)+D_{P}\left(w_{0}, u\right) \\
= & \int_{0}^{T}\left[\sum_{i=1}^{M} h\left(u_{i}(t) w_{i}(t)\right)+\sum_{i=1}^{M} g\left(w_{i}(t)\right)\right. \\
& \left.+\sum_{i=1}^{M} \sum_{j=1}^{M} A_{i j} f_{i j}(t) \alpha_{i}(t) d_{i j}\right] \mathrm{d} t .
\end{aligned}
$$

For convenience, let the utility function be

$$
\begin{aligned}
U(w(t), u(t))= & \sum_{i=1}^{M} h\left(u_{i}(t) w_{i}(t)\right)+\sum_{i=1}^{M} \sum_{j=1}^{M} A_{i j} f_{i j}(t) \alpha_{i}(t) d_{i j} \\
& +\sum_{i=1}^{M} g\left(w_{i}(t)\right) .
\end{aligned}
$$

The goal of this paper is to find an optimal dynamic CA policy $u \in \Omega$ with the constrain of the proposed workload model (1), so that the overall latency $J$ is minimized. Therefore, we can formulate an optimal control problem as follows.

$$
\begin{gathered}
\min _{u \in \Omega} J\left(w_{0}, u\right)=\int_{0}^{T} U(w(t), u(t)) \mathrm{d} t \\
\text { s.t. }\left\{\begin{array}{c}
\dot{w}_{i}(t)=\alpha_{i}(t) f_{i i}(t)+\sum_{j=1}^{M} A_{j i} f_{j i}(t) \alpha_{j}(t)-u_{i}(t) w_{i}(t), \\
0 \leq t \leq T, i=1,2, \ldots, M, \\
w(0)=w_{0},
\end{array}\right.
\end{gathered}
$$


For convenience, we refer to this optimal control problem as the Optimal Cloud Assistance problem.

\section{Solution}

In the previous subsection, we formulated an continuoustime optimal control problem (12) for seeking optimal CA policies, so that the overall latency is minimized. In this subsection, we now move on to solving the formulated optimal control problem.

As the problem is time-continuous, Pontryagin Minimum Principle [21] provides an effective approach to solving it. As an outline, Pontryagin Minimum Principle gives a set of necessary conditions for optimal solutions to an optimal control problem, which we refer to as the optimality system. By solving the system, we are able to find the most likely ones to be optimal controls. To find controls that satisfy all the necessary conditions in the optimality system, we apply an iterative algorithm known as the Forward-Backward Sweep Method [22]. Finally, we recommend the obtained optimal solution as an optimal CA policy.

\section{Optimality system}

Let us discuss the necessary conditions of optimal solutions. We begin by formulating the Hamiltonian function for the collaborative offloading problem (12).

$$
\begin{aligned}
H(w, u, p) & =U(w, u)+p^{T} \dot{w} \\
& =\sum_{i=1}^{M} h\left(u_{i} w_{i}\right)+\sum_{i=1}^{M} \sum_{j=1}^{M} A_{i j} f_{i j} \alpha_{i} d_{i j}+\sum_{i=1}^{M} g\left(w_{i}\right) \\
& +\sum_{i=1}^{M} p_{i}\left(-u_{i} w_{i}+\alpha_{i} f_{i i}+\sum_{j=1}^{M} A_{j i} f_{j i} \alpha_{j}\right)
\end{aligned}
$$

where $p=\left(p_{1}, \ldots, p_{M}\right)^{T}$ is the adjoint vector of the Hamiltonian function.

Denote by $u(t) \in \Omega$ an optimal control, $w(t)$ the associated workload distribution, and $p(t)$ the associated adjoint function. Then, combining the statement in Pontryagin Minimum Principle, we give the following theorem that shows a set of necessary conditions to optimal controls.

Theorem 2 The optimal control $u(t)$, optimal workload distribution trajectory $w(t)$, and adjoint function $p(t)$ must satisfy the following two conditions simultaneously.

1 The optimal control $u(t)$ must meet the condition that

$$
u_{i}(t)=\arg \min _{z \in[0,1]} h\left(z w_{i}\right)-p_{i} w_{i} z, \quad 0 \leq t \leq T, i=1,2, \ldots, M
$$

2 The optimal workload distribution trajectory $w(t)$ evolves according to the workload model (1), and adjoint function $p(t)$ evolves according to

$$
\left\{\begin{aligned}
\dot{p}_{i}(t)= & {\left[p_{i}(t)-h^{\prime}\left(u_{i}(t) w_{i}(t)\right)\right] u_{i}(t)-g^{\prime}\left(w_{i}(t)\right) } \\
& -\sum_{j=1}^{M} p_{j}(t) \alpha_{j}(t) \frac{\partial f_{j j}}{\partial w_{i}}(t) \\
& -\sum_{j=1}^{M} \sum_{k=1}^{M} A_{j k} \alpha_{j}(t) d_{j k} \frac{\partial f_{j k}}{\partial w_{i}}(t) \\
& -\sum_{j=1}^{M} p_{j}(t) \sum_{k=1}^{M} A_{k j} \alpha_{k}(t) \frac{\partial f_{k j}}{\partial w_{i}}(t) \\
& 0 \leq t \leq T, i=1,2, \ldots, M \\
p(T)= & 0 .
\end{aligned}\right.
$$

Proof According to Pontryagin Minimum Principle, for all admissible controls $u^{\prime}(t) \in \Omega$, it follows that

$$
H(w(t), u(t), p(t)) \leq H\left(w(t), u^{\prime}(t), p(t)\right), \forall t \in[0, T]
$$

In particular, let

$$
\Phi(u)=\sum_{i=1}^{M} h\left(u_{i} w_{i}\right)-\sum_{i=1}^{M} p_{i} w_{i} u_{i}
$$

which is refined from the Hamiltonian function by removing coefficients. Because all elements of $u$ are independent on each other, when $t$ is given it follows that

$$
u_{i}(t)=\arg \min _{z \in[0,1]} h\left(z w_{i}\right)-p_{i} w_{i} z, \quad i=1,2, \ldots, M
$$

Besides, by applying Pontryagin Minimum Principle again, it follows that

$$
\left\{\begin{array}{l}
\dot{w}_{i}(t)=+\frac{\partial H}{\partial p_{i}}(w(t), u(t), p(t)), \quad 0 \leq t \leq T, i=1,2, \ldots, M, \\
w(0)=w_{0} \\
\dot{p}_{i}(t)=-\frac{\partial H}{\partial w_{i}}(w(t), u(t), p(t)), \quad 0 \leq t \leq T, i=1,2, \ldots, M, \\
p(T)=0 .
\end{array}\right.
$$

By calculation, we can get the above results. The proof is completed.

The above two conditions shape the characteristics of optimal controls. By solving the formulated optimality system, we are able to find all possible optimal solutions to the optimal cloud assistance problem (12). 


\section{Further properties of optimal solutions}

In the previous subsection, we derived the optimality system for seeking optimal solutions to the optimal cloud assistance problem (12), in which a sub-problem (14) needed to be solved.

Because the sub-problem is a bounded one-dimensional optimization problem, we can solve the problem by numerical searching. However, the searching can sometimes be time-consuming. By observing the sub-problem (14), we find that if the function $h$ is continuous, the convexity and concavity of the function $q(z)=h\left(z w_{i}\right)-$ $p_{i} w_{i} z$ are dependent on those of the function $h$. By using this property, we can help accelerate solving the subproblem (14) in some cases. The conclusions are given below.

As the first case, when the function $h$ is convex, we can get the following conclusion.

Theorem 3 If the function $h$ is continuous and convex, any local minimum of the function $q$ is the global minimum of $q$ as well. Moreover, if the function $h$ is once continuously differentiable, it follows that: (a) if $h^{\prime}\left(w_{i}(t)\right) w_{i}(t)-p_{i}(t) w_{i}(t) \leq 0$, then $u_{i}(t)=1$; (b) if $h^{\prime}(0) w_{i}(t)-p_{i}(t) w_{i}(t) \geq 0$, then $u_{i}(t)=$ 0 ; (c) otherwise, $u_{i}(t)$ is the solution of the equation $h^{\prime}\left(u_{i}(t) w_{i}(t)\right) w_{i}(t)-p_{i}(t) w_{i}(t)=0$.

Proof As the function $h$ is convex, the function $q$ is convex as well. As a result, it follows from the Convexity Theory [50] that a local minimum of $q$ must be the global minimum of $q$. Moreover, if $q^{\prime}(0) \geq 0$, then $q^{\prime}(z) \geq 0, \forall z \in$ $[0,1]$, and therefore the minimum of $q$ must be obtained at $z=0$. If $q^{\prime}(0) \leq 0$, then $q^{\prime}(z) \leq 0, \forall z \in[0,1]$, and therefore the minimum of $q$ must be obtained at $z=1$. The proof is completed.

Theorem 3 shows that if the function $h$ is once continuously differentiable and convex, the component of the optimal control (i.e., $\left.u_{i}(t)\right)$ may be a binary policy. This property can sometimes accelerate solving the optimal CA policy when $u_{i}(t)$ has a binary structure, because in that case we have no need to use the one-dimensional numerical searching method for solving the sub-problem (14).

As the second case, when the function $h$ is concave, we can get the following conclusion.

Theorem 4 If the function $h$ is continuous and concave, it follows that: (a) if $h(0) \leq h\left(w_{i}(t)\right)-p_{i}(t) w_{i}(t)$, then $u_{i}(t)=0$; otherwise, $u_{i}(t)=1$.

Proof As the function $h$ is concave, the function $q$ is concave as well. As the result, the minimum of $q$ must be obtained at $\{0,1\}$ according to the values of $q(0)$ and $q(1)$.
Theorem 4 shows that if the function $h$ is continuous and concave, any component of the optimal control (i.e., $\left.u_{i}(t)\right)$ must be a binary policy. This property will greatly accelerate solving the optimal CA policy, because we can simply compare the objective values of $u_{i}(t)=0$ and $u_{i}(t)=1$ when solving the subproblem (14).

\section{Iterative algorithm}

In the previous subsections, we derived an optimality system and discussed its properties. Now, we discuss how to find such a control that satisfies such a system. Because solving an optimality system essentially requires solving a two-point boundary value problem [51], the forwardbackward sweep method (FBSM) [22] provides a practical approach. Based on the FBSM, we provide a specific algorithm (Algorithm 1), denoted by the optimal cloud assistance algorithm. In addition, we should mention that it is extremely difficult if not impossible to prove the convergence of this algorithm. An illustration of this difficulty can be found in [52]. Nevertheless, as reported in [53], the FBSM can be quickly run and shows a good convergence in general practical cases. Hence, we can still adopt the optimal cloud assistance algorithm. Moreover, it is difficult to find a previous theoretical analysis of the complexity of the FBSM method. We also had difficulty conducting such an analysis, and therefore we discuss the practical complexity of the algorithm through our numerical experiments.

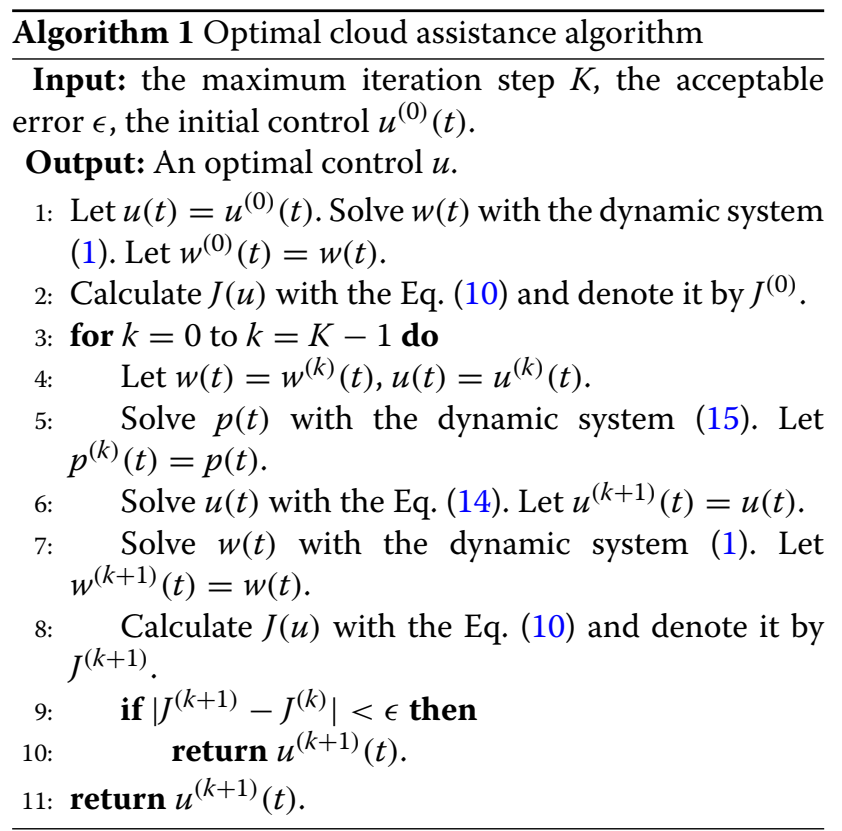

In lines 5 and 7 of Algorithm 1, we need to solve two dynamic systems characterized by ordinary differential equations. To solve ordinary differential equations, 
it is possible to apply numerical methods such as the Euler [54] and Runge-Kutta [55] methods. The RungeKutta approach has been proven to be more accurate but more time-consuming than the Euler method owing to its complexity. Nevertheless, when the step length is small enough, the difference between the two methods is generally negligible.

The relations among the FBSM, the optimal cloud assistance algorithm, the Euler method, and the RungeKutta method are as follows: (a) the implementation of the FBSM is dependent on methods such as the Euler and Runge-Kutta approaches used for solving ordinary differential equations, and (b) the optimal cloud assistance algorithm is a practical application of the FBSM.

\section{Illustrative examples}

In the previous section, we discussed how to solve the optimal cloud assistance problem. In this section, we provide several numerical examples to illustrate the problem and its optimal policies.

The remainder of this paper proceeds as follows. First, we provide a description of the experimental environment. Second, we define some common experimental settings used in our study, including the topological structure of the edge computing network and the workload computation delay function $g$. We then introduce two numerical examples and provide a brief analysis of the results obtained. Next, we discuss some common phenomena shown in the numerical examples. Finally, we investigate the convergence and complexity of the practical algorithm.

\section{Experimental environment}

First, let us start with a description of the experimental environment, including the hardware environment, the use of parameter values, and the programming language used in the experiments. All numerical experiments described in this study were conducted on a PC machine with an Intel i3 9100F CPU and 16 GB of RAM. In addition, the parameter values involved in all experiments were determined according to our experience. In fact, some parameters, such as the capacity of an edge server, the increased traffic rate of a DDoS attack, and the communication delays between two servers, are dependent on specific scenarios, and thus it can be difficult to find the standard values for them. For this reason, we set the parameter values according to our experience, with the purpose of investigating the influences of optimal CA policies. Some existing studies also set the parameter values according to experience. See [41, 43, 56] for certain examples. Moreover, all numerical experiments were implemented using the $\mathrm{C}++$ and Java programming languages.

\section{Common setup}

Let us move on to the topological structure of an edge computing network. Considering that there are $M=10$ ESs labeled by $1,2, \ldots, 10$, as shown in Fig 3. Each server has the same capacity of at most $10 \mathrm{k}$ tasks being processed simultaneously. Then, suppose that the overall computation delay function of server workload is defined by

$$
g(x)=g_{\max } \cdot\left(1-\frac{1}{1+e^{3(x-10)}}\right)
$$

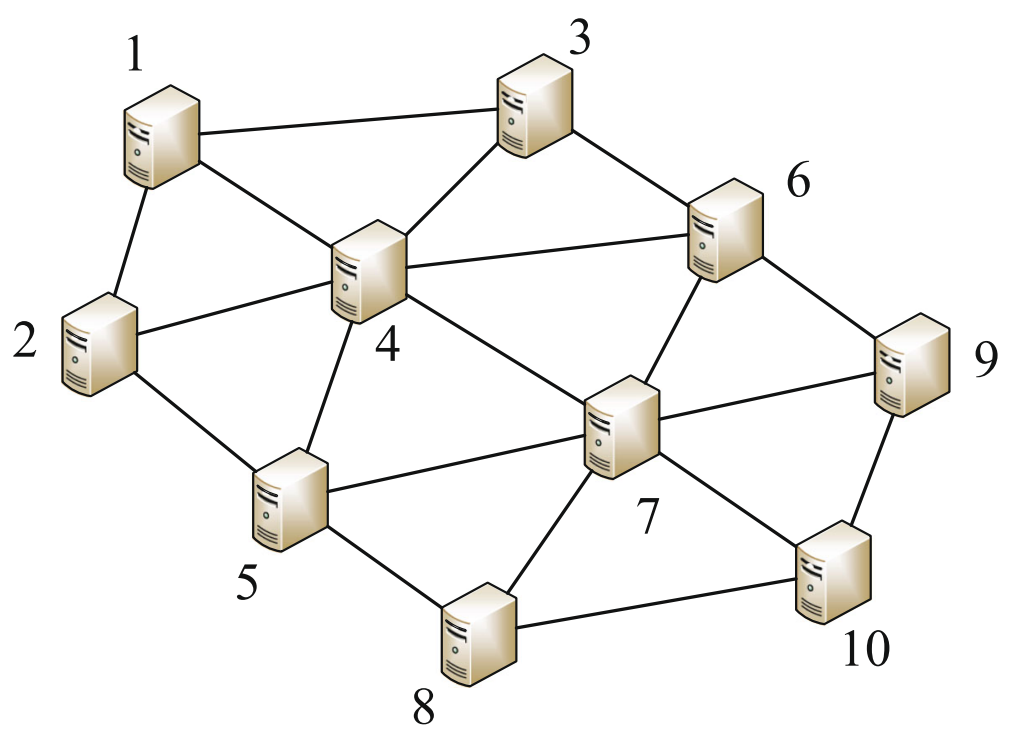

Fig. 3 Diagram of the topological structure of the distributed edge computing system 
where $g_{\max }$ is a constant coefficient. Figure 4 illustrates an instance of function $g(x)$. This function implies that the overall computation delay is negligible when the workload of a server is much less than its capacity, but will increase sharply to a maximum of $g_{\max }$ if the workload approaches capacity.

In the following two examples, we assume that the communication delay increases the function $h$ as a linear function. This assumption is reasonable. For example, if it takes an average of $0.5 \mathrm{~s}$ to transmit one task from an edge server to the cloud, it will take an average of $1 \mathrm{~s}$ to transmit two tasks.

To validate the optimal policies obtained, we use the allone policy $u^{(1)}(t) \equiv 1$ and the all-zero policy $u^{(0)}(t) \equiv 0$ as reference objects for the comparative experiments. The all-zero policy is used to investigate the situation in which there is no cloud assistance. The all-one policy is used to investigate the situation in which every edge server will always upload all pending tasks onto the cloud center. For convenience, we denote by $w^{(0)}(t)$ the workload distribution evolution function under the all-zero policy and $w^{(1)}(t)$ under the all-one policy. Moreover, we denote the obtained optimal policy by $u^{*}(t)$, and denote the corresponding workload distribution evolution function by $w^{*}(t)$.

\section{Examples, results, and explainations}

Having discussed the common experimental settings, we now introduce two numerical examples. To gain insight into the influences of different load balance schemes, the first example considers the RR scheme, and the second considers the LC scheme.

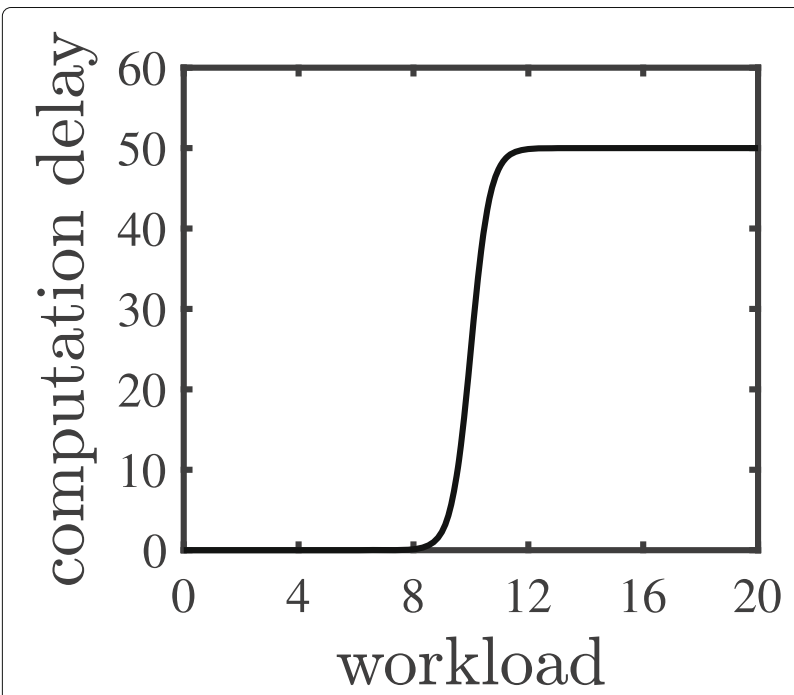

Fig. 4 An instance of the computation delay function $g(x)$, where $g_{\max }=50$
Example 1 Consider the situation that the total time horizon $T=5$ minutes $(m)$, the initial workload $w(0)=0$ tasks, the task increasing rate $\alpha(t) \equiv$ $(0,0,0,5,0,0,10,0,0,0)$ thousand tasks per minute $(\mathrm{kt} / \mathrm{m})$, the overall computation delay function $g$ where $g_{\max }=$ 1000 seconds per minute $(\mathrm{s} / \mathrm{m})$, the task forwarding ratio function $f_{i j}=f_{i j}^{R R}$, the cloud assistance communication delay function $h(z)=z$ seconds per thousand tasks $(s / k t)$, and the task forwarding communication delay $d_{i j}=0.3$ seconds per thousand tasks ( $s / k t)$.

Results: The results of Example 1 are shown in Figs. 5, 6, and 7. Figure 5 shows the optimal policy. From Fig. 5, we can see that the related assistance rates of servers $s_{4}$, $s_{5}, s_{6}$, and $s_{7}$ maintain the maximum during the entire time horizon, whereas those of the other servers maintain the minimum. Moreover, as the function $h$ has a concave property, it follows from Theorem 4 that the optimal policy has a binary structure. Figure 6 compares the workload distribution evolution under the optimal policy, the all-zero policy, and the all-one policy. Figure $7 \mathrm{com}$ pares the accumulated overall latency under the optimal policy, the all-zero policy, and the all-one policy. From Figs. 6 and 7, we can see the influence of the different policies:

- Under the all-zero policy, the workload of servers $s_{1}$, $s_{2}$, and $s_{3}$ increases linearly from zero to $4 \mathrm{k}$ tasks, the workload of servers $s_{8}, s_{9}$, and $s_{10}$ increases linearly from zero to $7 \mathrm{k}$ tasks, and the workload of servers $s_{4}$, $s_{5}, s_{6}$, and $s_{7}$ increases linearly from zero to more than $10 \mathrm{k}$ tasks. As a result, the overall latency increases slowly for a long time after the beginning, but then increases dramatically to an unacceptable value of nearly $1.5 \mathrm{k} \mathrm{s}$ owing to the high workload of servers $s_{4}, s_{5}, s_{6}$, and $s_{7}$.

- Under the all-one policy, all servers enjoy low-level workload over the entire time horizon. As a result, the overall latency enjoys a low level of approximately $80 \mathrm{~s}$.

- Under the optimal policy, the workload of servers $s_{1}$, $s_{2}$, and $s_{3}$ increases linearly to a low level of $4 \mathrm{k}$ tasks, the workload of servers $s_{4}, s_{5}, s_{6}$, and $s_{7}$ increases slowly to a low level of $2 \mathrm{k}$ tasks, and the workload of servers $s_{8}, s_{9}$, and $s_{10}$ increases linearly to a middle level of $7 \mathrm{k}$ tasks. As a result, the overall latency enjoys a low level of nearly $50 \mathrm{~s}$, which is $37.5 \%$ less than that of the all-one policy, and even much less than that of the all-zero policy.

From the above observations, we can conclude the following:

- If the all-zero policy is adopted, there will be a high computation delay owing to the high server workload. 

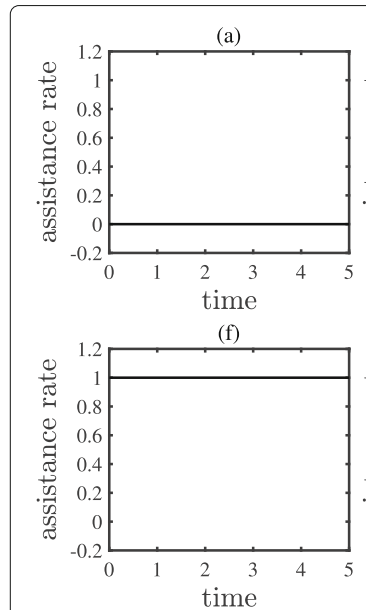

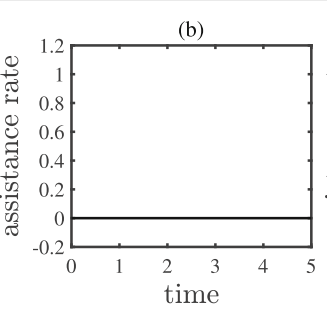

(g)

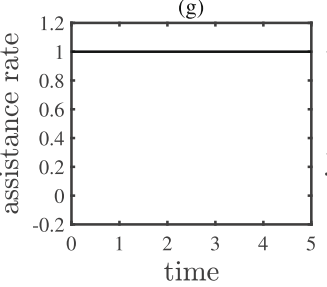

(c)

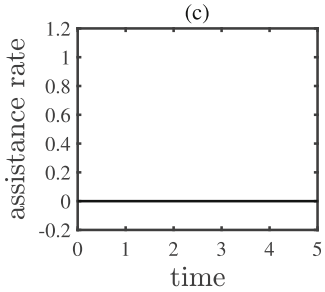

(h)

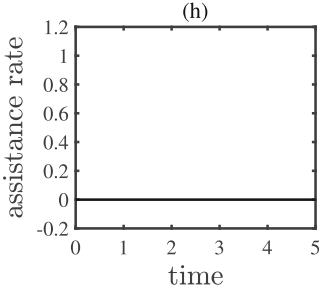

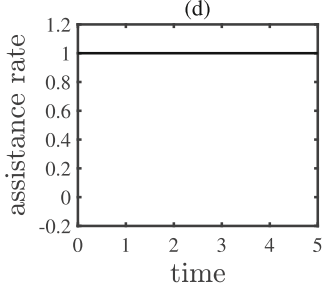

(i)

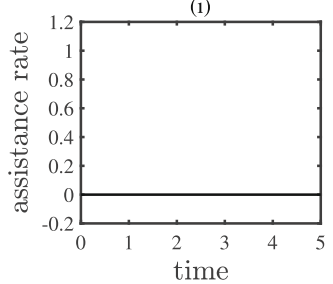

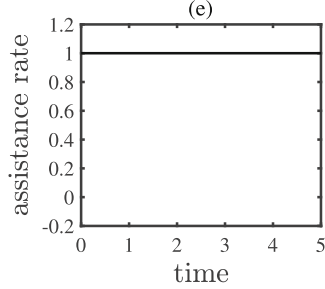

(j)

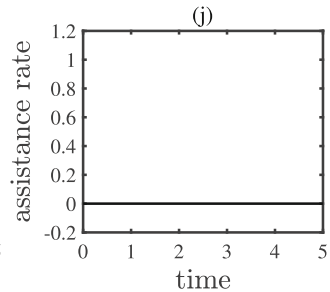

Fig. 5 The optimal policy obtained from Example 1 , where $(\mathbf{a})$ to $(\mathbf{j})$ show the elements $u_{1}(t), u_{2}(t), \ldots, u_{10}(t)$ in order

- If the all-one policy is adopted, there will be a high communication delay owing to the long distance between the edge and cloud sides.

- If the optimal policy is adopted, there will be a trade-off between the computation delay and communication delay, and thus the overall latency will be minimized.

Therefore, the obtained optimal policy is effective.

Reasons: When servers $s_{4}$ and $s_{7}$ are under attack, the workload of servers $s_{4}$ and $s_{7}$ will increases sharply and reach an intolerable level. In addition, because servers $s_{5}$ and $s_{6}$ are connected directly to both servers $s_{4}$ and $s_{7}$, and because the RR workload balance scheme will spread newly increased traffic among the connected servers, the workload of servers $s_{5}$ and $s_{6}$ will also quickly increase (more slowly than those of servers $s_{4}$ and $s_{7}$ ) and reach an intolerable level. The remaining servers (i.e., $s_{1}, s_{2}, s_{3}, s_{8}$, $s_{9}$, and $s_{10}$ ) are only connected to one of the servers, $s_{4}$ or $s_{7}$, and thus the workload of the remaining servers will increase at a relatively low rate within a tolerable range. To maintain the overall workload at a low level without introducing excessive communication latency, the servers $s_{4}, s_{5}, s_{6}$, and $s_{7}$ need to upload pending tasks to the cloud center at a high rate, whereas the remaining servers only need to handle the tasks with their own capacity to avoid unnecessary communication delays. If not, the EEC ecosystem will suffer high communication delays or high computation delays because the servers cannot cooperate effectively and make the best trade-off.

Example 2 Consider the situation that the total time horizon $T=5$ minutes $(m)$, the initial workload $w(0)=0$ tasks, the task increasing rate $\alpha(t) \equiv$ $(0,0,0,5,0,0,10,0,0,0)$ thousand tasks per minute $(\mathrm{kt} / \mathrm{m})$, the overall computation delay function $g$ where $g_{\max }=$
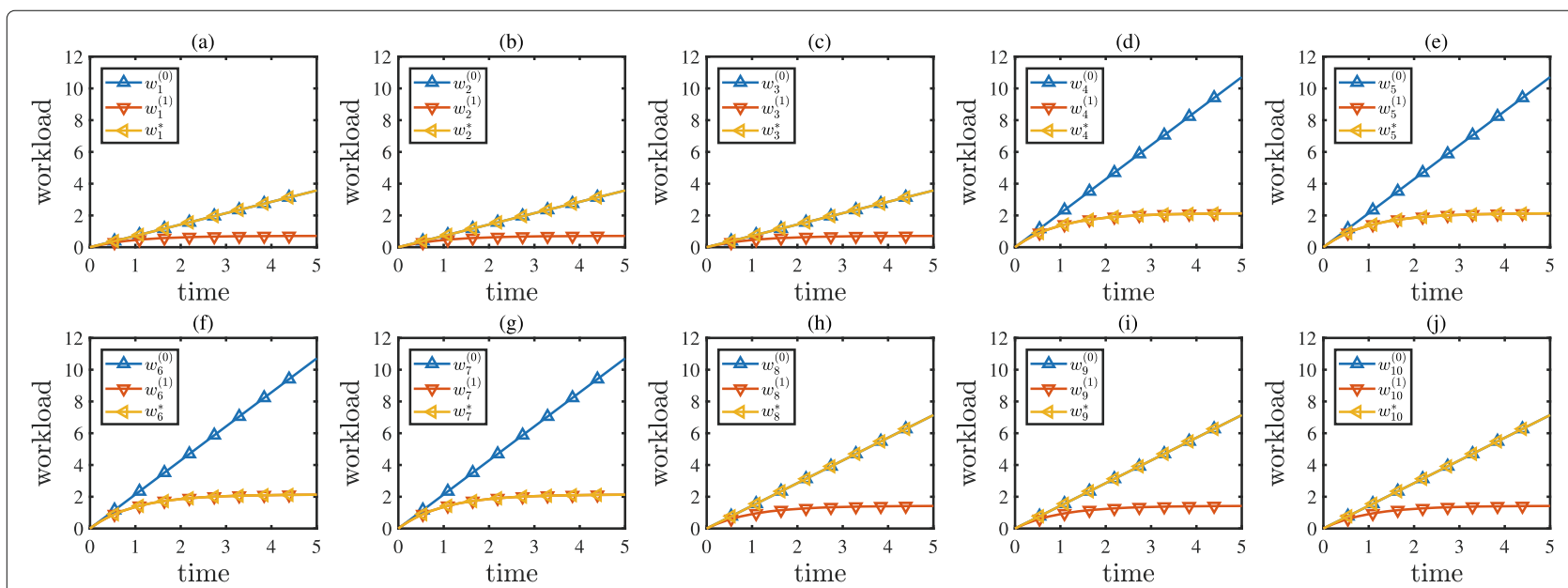

Fig. 6 The workload distribution evolution obtained from Example 1, where (a) to (j) show the workload dynamics of servers $s_{1}, s_{2}, \ldots, s_{10}$ in order 

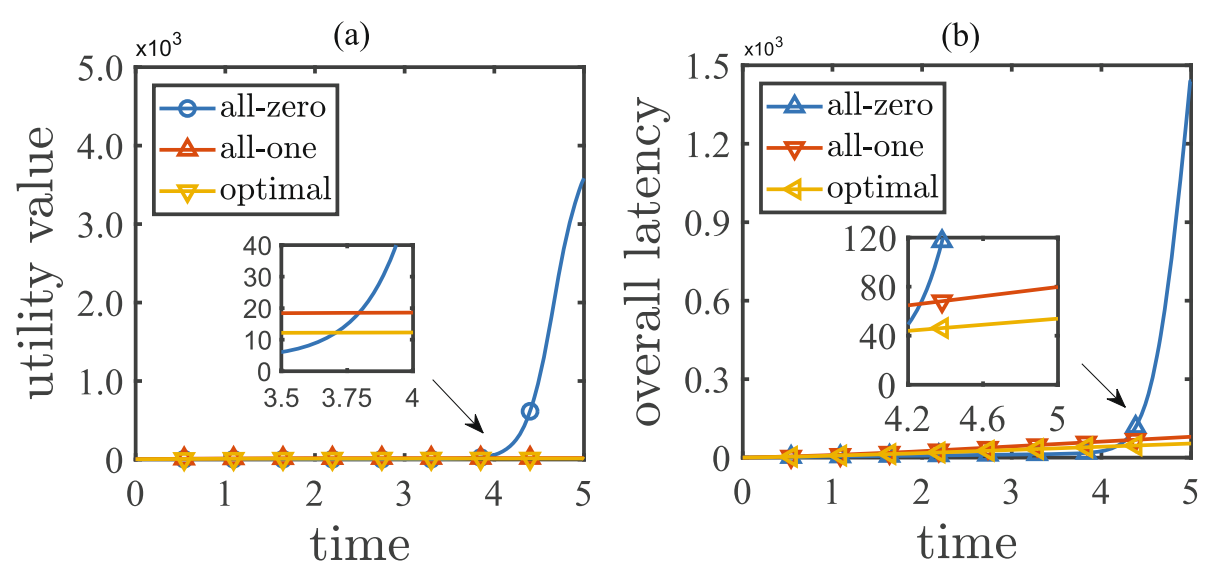

Fig. 7 Performance results of Example 1: (a) the utility function over time; (b) the accumulated overall latency over time

1000 seconds per minute ( $\mathrm{s} / \mathrm{m})$, the task forwarding ratio function $f_{i j}=f_{i j}^{L C}$ where $\epsilon=0.001$, the cloud assistance communication delay function $h(z)=z$ seconds per thousand tasks ( $s / k t)$, and the task forwarding communication delay $d_{i j}=0.3$ seconds per thousand tasks $(\mathrm{s} / \mathrm{kt})$.

Results: The results of Example 2 are shown in Figs. 8, 9, and 10. Figure 8 shows the optimal policy. From Fig. 8 , we can see that all related assistance rates maintain the maximum for a period of time after the beginning, then drop to and maintain the minimum before the termination time. Moreover, as function $h$ has a concave property, it follows from Theorem 4 that the optimal policy has a binary structure. Figure 9 compares the workload distribution evolution under the optimal policy, the all-zero policy, and the all-one policy. Figure 10 compares the accumulated overall latency under the optimal policy, the all-zero policy, and the all-one policy. From Figs. 9 and 10, we can see the influence of different policies.

- Under the all-zero policy, the workload of servers $s_{1}$, $s_{2}$, and $s_{3}$ increases linearly from zero to $5 \mathrm{k}$ tasks, the workload of servers $s_{8}, s_{9}$, and $s_{10}$ increases linearly from zero to $8 \mathrm{k}$ tasks, and the workload of servers $s_{4}$, $s_{5}, s_{6}$, and $s_{7}$ increases linearly from zero to more than $9 \mathrm{k}$ tasks. As a result, the overall latency increases slowly for a long time after the beginning, but then increases dramatically to nearly $90 \mathrm{~s}$ owing to the high workload of servers $s_{4}, s_{5}, s_{6}, s_{7}, s_{8}, s_{9}$, and $s_{10}$.

- Under the all-one policy, all servers enjoy low-level workload over the entire time horizon. As a result, the overall latency increases to nearly $80 \mathrm{~s}$ at the terminated time owing to unnecessary cloud assistance.

- Under the optimal policy, the workload of all servers first increases slowly for a period of time after the beginning and then increase linearly before the termination time. At the termination time, the workload of servers $s_{1}, s_{2}$, and $s_{3}$ enjoys a low level of $4.5 \mathrm{k}$ tasks, the workload of servers $s_{8}, s_{9}$, and $s_{10}$ is at a middle level of $7 \mathrm{k}$ tasks, and the workload of servers $s_{4}, s_{5}, s_{6}$, and $s_{7}$ is at a high level of $8.5 \mathrm{k}$. As a result, the overall latency enjoys a low level of $35 \mathrm{~s}$, which is $61.1 \%$ less than that under the all-zero policy, and $56.3 \%$ less than that under the all-one policy.

From the above observations, we can conclude the following:

- If the all-zero policy is adopted, there will be a high computation delay owing to the high server workload.

- If the all-one policy is adopted, there will be a high communication delay owing to the long distance between the edge and cloud sides.

- If the optimal policy is adopted, there will be a trade-off between the computation delay and communication delay, and thus the overall latency will be minimized.

Therefore, the obtained optimal policy is effective.

Reasons: When servers $s_{4}$ and $s_{7}$ are under attack, the workload of the servers $s_{4}$ and $s_{7}$ will increases sharply. In addition, because the servers $s_{5}$ and $s_{6}$ are connected directly to both servers $s_{4}$ and $s_{7}$, and because the LC workload balance scheme will rapidly spread newly increased traffic among connected servers, the workload of the servers $s_{5}$ and $s_{6}$ will also increase quickly. Although the remaining servers (i.e., $s_{1}, s_{2}, s_{3}, s_{8}, s_{9}, s_{10}$ ) are only connected to one of the servers $s_{4}$ and $s_{7}$, the workload of the remaining servers will increase at a relatively low but still high rate. Initially, to maintain the overall workload at a low level, all servers need to upload pending tasks to the cloud center at a high rate. After a while, all servers appear to know that if they continue to keep the cloud assistance rate at a high level, too much 

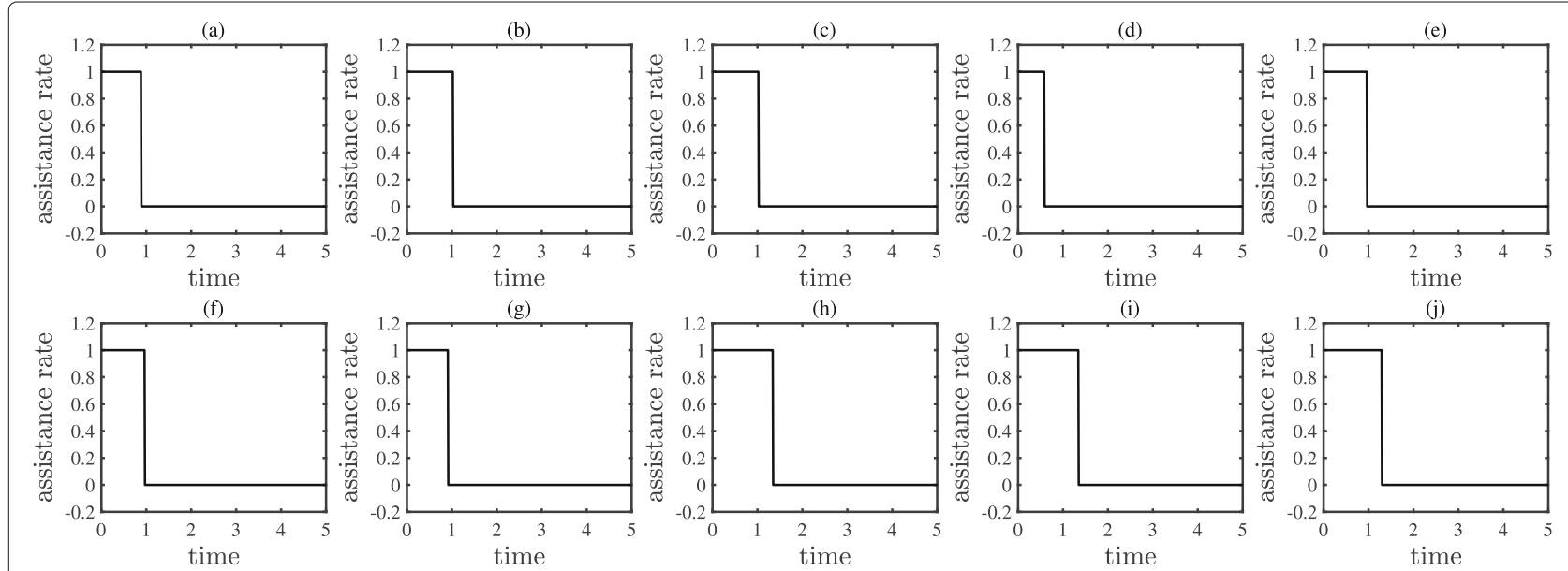

Fig. 8 The optimal policy obtained from Example 2, where $(\mathbf{a})$ to $(\mathbf{j})$ show the elements $u_{1}(t), u_{2}(t), \ldots, u_{10}(t)$ in order
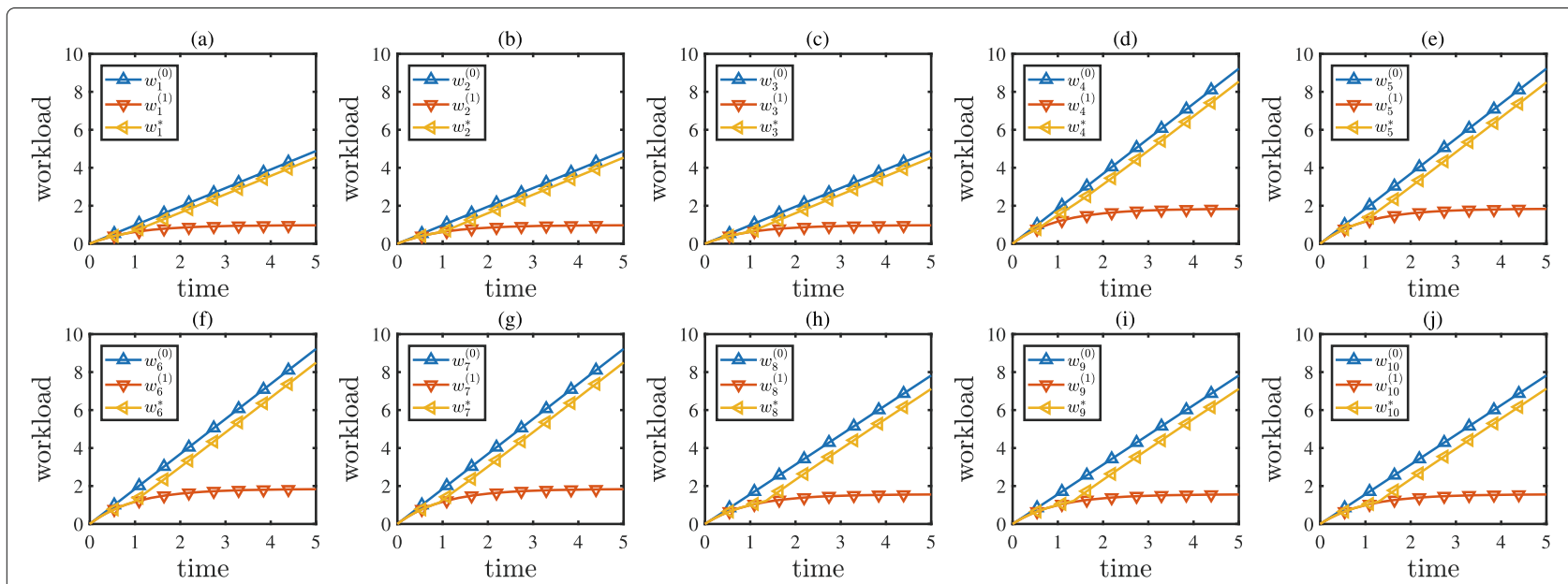

Fig. 9 The workload distribution evolution obtained from Example 2, where (a) to (j) show the workload dynamics of servers $s_{1}, s_{2}, \ldots, s_{10}$ in order
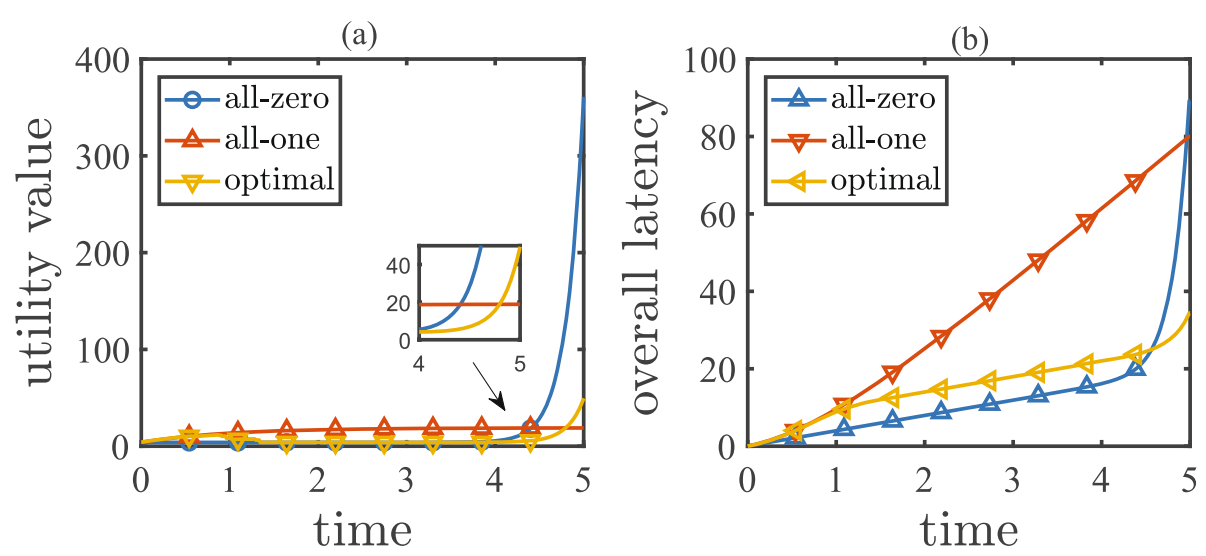

Fig. 10 Performance results of Example 2: (a) the utility function over time; (b) the accumulated overall latency over time 
communication latency will be introduced. All servers also find that, despite not uploading any pending tasks to the cloud center, they can still absorb all attacking traffic using their remaining computation capacity. Although doing this will arguably increase the computation latency, the computation latency introduced is still lower than the communication latency incurred by all servers while maintaining high cloud assistance rates. To achieve the best trade-off, all servers decide not to upload pending tasks after a certain timestamp.

\section{Discussions}

In this subsection, we discuss the role of workload balance in mitigating edge DDoS attacks, the effectiveness of the EEC ecosystem in absorbing undetectable malicious traffic, and the influence of the DDoS attack on the overall latency.

From Figs. 6 and 9, we can see the role of workload balance. The two aforementioned numerical examples consider the same attack scenario, where only servers $s 4$ and $s 7$ are under attack. As shown in the experimental parameters, there are $75 \mathrm{k}$ malicious tasks hitting servers $s_{4}$ and $s_{7}$ during an attack. When applying the all-zero policy, no pending tasks will be uploaded onto the cloud, and all tasks will be processed in the edge computing network. Without a load balance, the servers $s_{4}$ and $s_{7}$ will have to handle all $75 \mathrm{k}$ attacking tasks, and their workload will become extremely high. With a load balance, idle edge servers can help handle a portion of the attack traffic. Figure 6 shows that the $75 \mathrm{k}$ malicious tasks at $s_{4}$ and $s_{7}$ will be non-uniformly dispatched among the edge computing network, which is determined by the characteristics of the RR scheme. Figure 9 shows that the $75 \mathrm{k}$ malicious tasks at $s_{4}$ and $s_{7}$ are relatively uniformly dispatched among the edge computing network, which is determined by the characteristics of the LC scheme. It seems that the LC scheme is more efficient than the RR scheme because the
LC scheme can make the edge computing network absorb more traffic.

In addition, Figs. 6, 7, 9, and 10 show the effectiveness of an EEC ecosystem in absorbing undetectable malicious requests. When the edge computing network encounters a DDoS attack and malicious requests are undetectable, firewalls cannot filter out these abnormal requests, and the attacking traffic directly hits the target of the edge computing network. Without a cloud assistance of the EEC ecosystem, the edge workload distribution will evolve when applying the all-zero policy. The results show that the edge computing network cannot afford high-volume attack traffic. By contrast, with cloud assistance, resources can be efficiently allocated, and hence the overall latency can be dramatically reduced, as in the cases of applying the optimal policy and the all-one policy.

Moreover, by observing the cases of applying the all-zero policy, we can also see the influence of a DDoS attack. Without cloud assistance, all the attack traffic will be processed in the edge computing network, and the overall latency will only come from the communication delay of the load balance and the computation delays of the edge servers. From Fig. 7, we can see that, by applying the all-zero policy, the overall latency increases slowly before time $t=4$ but increases sharply after time $t=4$. This phenomenon is illustrated by the all-zero case in Fig. 6 . We can see that before time $t=4$, the workload of all edge servers is fewer than $8 \mathrm{k}$ tasks, and after time $t=4$, the workload of servers $s 4, s 5, s 6$, and $s 7$ is more than $8 \mathrm{k}$ tasks. The computation delay function plotted in Fig. 4 shows that when the workload of a server is more than $8 \mathrm{k}$ tasks, the computation delay increases rapidly. Hence, the overall latency increases significantly after time $t=4$. Figure 10 shows a similar phenomenon, where the overall latency increases linearly before time $t=4.5$ but increases exponentially after time $t=4.5$. By observing the all-zero case in Fig. 9, we can see that before time (a)

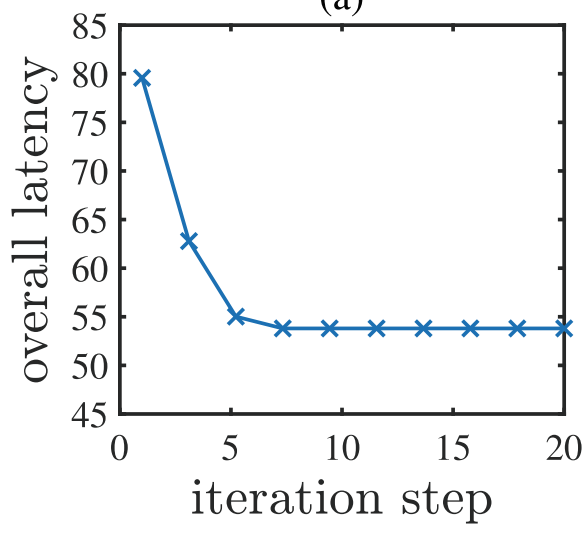

(b)

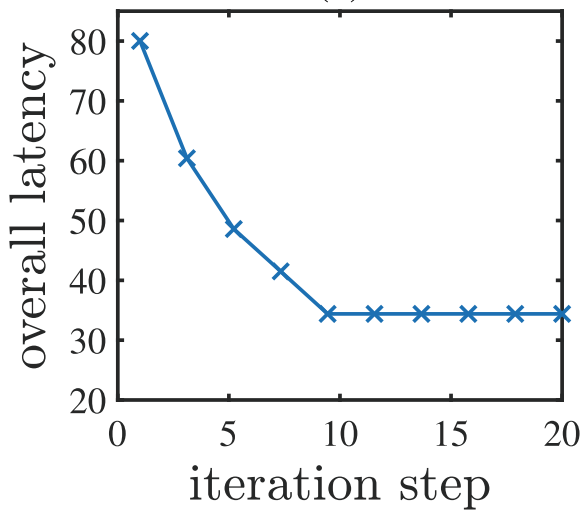

Fig. 11 Algorithm convergence: (a) the result of Example 1, (b) the result of Example 2 
(a)

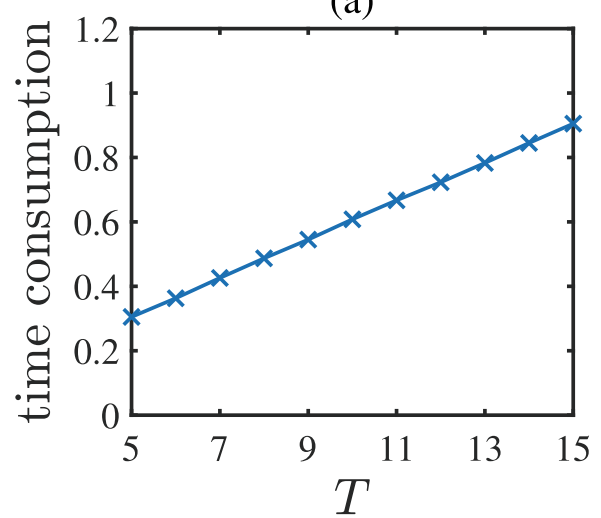

(b)

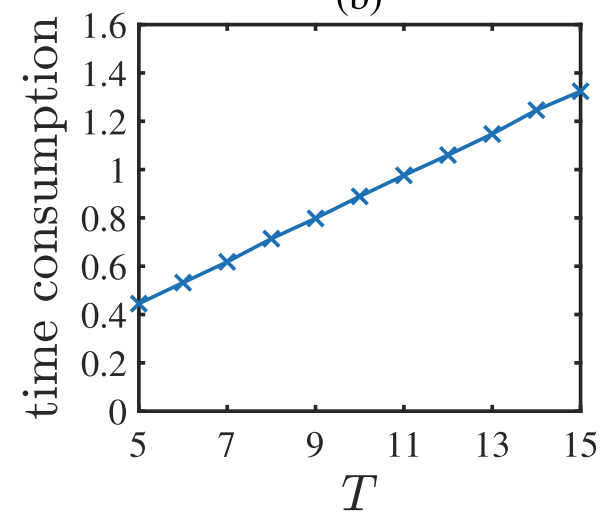

Fig. 12 Practical time consumption (seconds): (a) the result of Example 1, (b) the result of Example 2

$t=4.5$, all workload is fewer than $8 \mathrm{k}$ tasks, and after time $t=4.5$, the workload of servers $s 4, s 5, s 6$, and $s 7$ is more than $8 \mathrm{k}$ tasks. As a result, the computation delays of these edge servers increase rapidly. Therefore, we can conclude that, (a) initially, a DDoS attack will not seriously affect the overall latency because at that time the edge computing network can still handle the attack traffic itself, and (b) after a certain timestamp, the DDoS attack will significantly increase the overall latency because some edge servers in the network will have to suffer a high workload.

\section{Algorithm convergence and complexity}

Finally, let us investigate the algorithm convergence and complexity.

In Fig. 11, we show the algorithm convergence for each of the two numerical examples. From the results, we can see that the optimal cloud assistance algorithm (Algorithm 1) shows a good convergence. The objective functional (overall latency) can converge quickly with only several iteration steps, which matches the discussion in [53].

By observing the proposed optimal cloud assistance algorithm (Algorithm 1), we found that the termination time $T$ is an essential factor in the algorithm complexity. For each iteration step, we need to solve multiple ordinary differential equations and a one-dimensional optimization sub-problem. Given a method to solve the sub-problem, the overall algorithm complexity is mainly dependent on solving ordinary differential equations. If we set the same step length of time when numerically solving ordinary differential equations, the termination time $T$ determines the overall algorithm complexity.

Hence, to gain insight into the complexity of the algorithm, we investigate how the practical time consumption will increase as the termination time $T$ increases. Numerical experiments were conducted in the same hardware environment. In Fig. 12, we show the practical time consumption for each of the two numerical examples. From the results, we can see that the proposed optimal cloud assistance algorithm (Algorithm 1) runs quickly and shows a linear complexity for the terminated time $T$.

\section{Conclusion}

In this paper, the design of optimal cloud assistance policies for the use of an end-edge-cloud ecosystem to mitigate edge DDoS attacks is described. First, we propose an edge workload model that incorporates the edge server workload distribution evolution under a given cloud assistance policy. Based on the model, we quantify the effectiveness of the policy based on the resulting overall latency and formulate an optimal control problem for seeking optimal cloud assistance policies that can minimize the overall latency. Then, following the Pontryagin's minimum principle, we provide solutions by deriving an optimality system. To accelerate the problem solving, we also discuss some properties of the optimal solutions. Next, we introduce a numerical iterative algorithm based on the forward-backward sweep method to seek policies that satisfy the derived optimality system. Finally, we provide numerical examples to illustrate the optimal policies. Our work will assist in the development of a design for using an end-edge-cloud ecosystem to mitigate edge DDoS attacks.

\section{Acknowledgements \\ Not applicable.}

\section{Authors' contributions}

$T L$ is the only contributor of this paper. The author read and approved the final manuscript.

\section{Authors' information}

Teng Li received the B.Sc. degree from the School of Economics and Business Administration, Chongqing University, in 2006, and the M.Sc. degree from the School of Software Engineering, Chongqing University, in 2009. He is currently an Associate Professor with the Artificial Intelligence and Big Data College, 
Chongqing College of Electronic Engineering. His research interests include cloud computing, edge computing, and cyber security.

\section{Funding}

This research was funded by Chongqing Municipal Education Commission grant number KJ QN201903110.

\section{Availability of data and materials}

The data used during the current study are available from the corresponding author on reasonable request.

\section{Declarations}

\section{Competing interests}

The author declares that there is no competing interests.

\section{Received: 29 December 2020 Accepted: 18 July 2021}

Published online: 31 July 2021

\section{References}

1. Nord JH, Koohang A, Paliszkiewicz J (2019) The internet of things: Review and theoretical framework. Expert Syst Appl 133:97-108

2. Ray PP (2018) A survey on internet of things architectures. J King Saud Univ-Comput Inf Sci 30(3):291-319

3. Abbas N, Zhang Y, Taherkordi A, Skeie T (2017) Mobile edge computing: A survey. IEEE Internet Things J 5(1):450-465

4. Mao Y, You C, Zhang J, Huang K, Letaief KB (2017) A survey on mobile edge computing: The communication perspective. IEEE Commun Surv Tutor 19(4):2322-2358

5. Mahmood K, Li X, Chaudhry SA, Naqvi H, Kumari S, Sangaiah AK, Rodrigues JJ (2018) Pairing based anonymous and secure key agreement protocol for smart grid edge computing infrastructure. Futur Gener Comput Syst 88:491-500

6. Hossain MS, Muhammad G (2017) Emotion-aware connected healthcare big data towards 5G. IEEE Internet Things J 5(4):2399-2406

7. Shladover SE (2018) Connected and automated vehicle systems: Introduction and overview. J Intell Transp Syst 22(3):190-200

8. Zargar ST, Joshi J, Tipper D (2013) A survey of defense mechanisms against distributed denial of service (ddos) flooding attacks. IEEE Commun Surv Tutor 15(4):2046-2069

9. Osanaiye O, Choo K-KR, Dlodlo M (2016) Distributed denial of service (ddos) resilience in cloud: review and conceptual cloud ddos mitigation framework. J Netw Comput Appl 67:147-165

10. Kolias C, Kambourakis G, Stavrou A, Voas J (2017) Ddos in the iot: Mirai and other botnets. Computer 50(7):80-84

11. De Donno M, Dragoni N, Giaretta A, Spognardi A (2018) Ddos-capable iot malwares: Comparative analysis and mirai investigation. Secur Commun Netw 2018:1-30

12. Vlajic N, Zhou D (2018) lot as a land of opportunity for ddos hackers. Computer 51(7):26-34

13. Jia Y, Zhong F, Alrawais A, Gong B, Cheng X (2020) Flowguard: An intelligent edge defense mechanism against iot ddos attacks. IEEE Internet Things J 7:9552-9562

14. Liu J, Wang X, Shen S, Yue G, Yu S, Li M (2020) A bayesian q-learning game for dependable task offloading against ddos attacks in sensor edge cloud. IEEE Internet Things J 8:7546-7561

15. Xu Z, Liang W, Jia M, Huang M, Mao G (2018) Task offloading with network function requirements in a mobile edge-cloud network. IEEE Trans Mob Comput 18(11):2672-2685

16. Ren J, Zhang D, He S, Zhang Y, Li T (2019) A survey on end-edge-cloud orchestrated network computing paradigms: Transparent computing mobile edge computing, fog computing, and cloudlet. ACM Comput Surv (CSUR) 52(6):1-36

17. Hong Z, Chen W, Huang H, Guo S, Zheng Z (2019) Multi-hop cooperative computation offloading for industrial iot-edge-cloud computing environments. IEEE Trans Parallel Distrib Syst 30(12):2759-2774

18. Wang B, Wang C, Huang W, Song Y, Qin X (2020) A survey and taxonomy on task offloading for edge-cloud computing. IEEE Access 8:186080-186101
19. Khalil IM, Khreishah A, Azeem M (2014) Cloud computing security: a survey. Computers 3(1):1-35

20. Hashem IAT, Yaqoob I, Anuar NB, Mokhtar S, Gani A, Khan SU (2015) The rise of "big data" on cloud computing: Review and open research issues. Inf Syst 47:98-115

21. Donald EK (2004) Pontryagin's minimum principle and state inequality constrains. In: Donald EK (ed). Optimal Control Theory: An Introduction, 2nd edn. Dover Publications, New York. pp 227-239

22. Lenhart S, Workman JT (2007) Forward-backward sweep method. In: Etheridge AM, Gross LJ (eds). Optimal Control Applied to Biological Models, 2nd edn. CRC Press, Boca Raton. pp 49-56

23. Yu S, Zhou W, Doss R (2008) Information theory based detection against network behavior mimicking ddos attacks. IEEE Commun Lett 12(4):318-321

24. Xie Y, Yu S-Z (2008) Monitoring the application-layer ddos attacks for popular websites. IEEE/ACM Trans Netw 17(1):15-25

25. Wang J, Yang X, Long K (2011) Web ddos detection schemes based on measuring user's access behavior with large deviation. In: 2011 IEEE Global Telecommunications Conference-GLOBECOM 2011. IEEE, Piscataway. pp 1-5

26. Wen S, Jia W, Zhou W, Zhou W, Xu C (2010) Cald: Surviving various application-layer ddos attacks that mimic flash crowd. In: 2010 Fourth International Conference on Network and System Security. IEEE, Piscataway. pp 247-254

27. Ni T, Gu X, Wang H, Li Y (2013) Real-time detection of application-layer ddos attack using time series analysis. J Control Sci Eng 2013:1-6

28. Zhou W, Jia W, Wen S, Xiang Y, Zhou W (2014) Detection and defense of application-layer ddos attacks in backbone web traffic. Futur Gener Comput Syst 38:36-46

29. Ujjan RMA, Pervez Z, Dahal K, Bashir AK, Mumtaz R, González J (2020) Towards sflow and adaptive polling sampling for deep learning based ddos detection in sdn. Futur Gener Comput Syst 111:763-779

30. Hosseini S, Azizi M (2019) The hybrid technique for ddos detection with supervised learning algorithms. Comput Netw 158:35-45

31. Gao H, Tang M, Liu Y, Zhang P, Liu X (2017) Research on the security of microsoft's two-layer captcha. IEEE Trans InfForensics Secur 12(7):1671-1685

32. Tang M, Gao H, Zhang Y, Liu Y, Zhang P, Wang P (2018) Research on deep learning techniques in breaking text-based captchas and designing image-based captcha. IEEE Trans Inf Forensics Secur 13(10):2522-2537

33. Zi Y, Gao H, Cheng Z, Liu Y (2019) An end-to-end attack on text captchas. IEEE Trans Inf Forensics Secur 15:753-766

34. Wang P, Gao H, Rao Q, Luo S, Yuan Z, Shi Z (2020) A security analysis of captchas with large character sets. IEEE Trans Dependable Secure Comput. https://doi.org/10.1109/TDSC.2020.2971477

35. Kim K, You Y, Park M, Lee K (2018) Ddos mitigation: Decentralized cdn using private blockchain. In: 2018 Tenth International Conference on Ubiquitous and Future Networks (ICUFN). IEEE, Piscataway. pp 693-696

36. Imthiyas M, Wani S, Abdulghafor RAA, Ibrahim AA, Mohammad AH (2020) Ddos mitigation: A review of content delivery network and its ddos defence techniques. Int J Perceptive Cognitive Comput 6(2):67-76

37. Noh D-H, You D-Y, An B-K (2009) A solution method for ddos attack using cdn technology. J Inst Internet Broadcast Commun 9(2):91-96

38. Wang D, Chen D, Guo R (2013) Ddos mitigation in content distribution networks. Int J Wirel Mob Comput 6(5):508-514

39. Al-Qudah Z, Al-Duwairi B, Al-Khaleel O (2014) Ddos protection as a service: hiding behind the giants. Int J Comput Sci Eng 9(4):292-300

40. Li X, Ge X, Wang X, Cheng J, Leung VC (2016) Energy efficiency optimization: Joint antenna-subcarrier-power allocation in ofdm-dass. IEEE Trans Wirel Commun 15(11):7470-7483

41. You C, Huang K, Chae H, Kim B-H (2016) Energy-efficient resource allocation for mobile-edge computation offloading. IEEE Trans Wirel Commun 16(3):1397-1411

42. Wang Y, Sheng M, Wang X, Wang L, Li J (2016) Mobile-edge computing: Partial computation offloading using dynamic voltage scaling. IEEE Trans Commun 64(10):4268-4282

43. Mao Y, Zhang J, Letaief KB (2016) Dynamic computation offloading for mobile-edge computing with energy harvesting devices. IEEE J Sel Areas Commun 34(12):3590-3605

44. Zhang Z, Yu FR, Fu F, Yan Q, Wang Z (2018) Joint offloading and resource allocation in mobile edge computing systems: An actor-critic approach. In: 2018 IEEE Global Communications Conference (GLOBECOM). IEEE, Piscataway. pp 1-6 
45. Chen Y, Zhang N, Zhang Y, Chen X (2018) Dynamic computation offloading in edge computing for internet of things. IEEE Internet Things 」 6(3):4242-4251

46. Chen Y, Zhang N, Zhang Y, Chen X, Wu W, Shen XS (2019) Energy efficient dynamic offloading in mobile edge computing for internet of things. IEEE Trans Cloud Comput. https://doi.org/10.1109/TCC.2019.2898657

47. Zhang G, Zhang W, Cao Y, Li D, Wang L (2018) Energy-delay tradeoff for dynamic offloading in mobile-edge computing system with energy harvesting devices. IEEE Trans Ind Inf 14(10):4642-4655

48. Rasmussen RV, Trick MA (2008) Round robin scheduling-a survey. Eur J Oper Res 188(3):617-636

49. Teo YM, Ayani R (2001) Comparison of load balancing strategies on cluster-based web servers. Simulation 77(5-6):185-195

50. Bertsekas DP (1997) Nonlinear programming. J Oper Res Soc 48:334-334

51. Keller HB (2018) Numerical Methods for Two-point Boundary-value Problems. Courier Dover Publications, New York

52. McAsey M, Mou L, Han W (2012) Convergence of the forward-backward sweep method in optimal control. Comput Optim Appl 53:207-226

53. Lenhart S, Workman JT (2007) Optimal Control Applied to Biologica Models. CRC press, Boca Raton

54. Hoffman JD, Frankel S (2001) The first-order euler method. In: Frankel S (ed). Numerical Methods for Engineers and Scientists, 2nd edn. CRC Press, Boca Raton. pp 352-370

55. Butcher JC (2008) Runge-kutta methods. In: Butcher JC (ed). Numerical Methods for Ordinary Differential Equations, 2nd edn. Wiley, Hoboken. pp 97-108

56. Guo X, Singh R, Zhao T, Niu Z (2016) An index based task assignment policy for achieving optimal power-delay tradeoff in edge cloud systems. In: 2016 IEEE International Conference on Communications (ICC). IEEE, Piscataway. pp 1-7

\section{Publisher's Note}

Springer Nature remains neutral with regard to jurisdictional claims in published maps and institutional affiliations.

\section{Submit your manuscript to a SpringerOpen ${ }^{\circ}$ journal and benefit from:}

- Convenient online submission

- Rigorous peer review

- Open access: articles freely available online

- High visibility within the field

- Retaining the copyright to your article

Submit your next manuscript at $\gg$ springeropen.com 